\title{
Axial-Current Matrix Elements in Light Nuclei from Lattice QCD
}

\author{
Martin J. Savage*; Emmanuel Chang and Michael L. Wagman \\ Institute for Nuclear Theory, Seattle, Washington 98195-1550, USA. \\ E-mail: mjs5@uw. edu
}

\section{Silas R. Beane}

Department of Physics, University of Washington, Box 351560, Seattle, WA 98195, USA.

\section{Zohreh Davoudi, William Detmold and Phiala E. Shanahan}

Center for Theoretical Physics, Massachusetts Institute of Technology, Cambridge, MA 02139, USA.

\section{Kostas Orginos}

Department of Physics, College of William and Mary, Williamsburg, VA 23187-8795, USA and Jefferson Laboratory, 12000 Jefferson Avenue, Newport News, VA 23606, USA.

\section{Brian C. Tiburzi}

Department of Physics, The City College of New York, New York, NY 10031, USA and Graduate School and University Center, The City University of New York, New York, NY 10016, USA and RIKEN BNL Research Center, Brookhaven National Laboratory, Upton, NY 11973, USA.

\section{Frank Winter}

Jefferson Laboratory, 12000 Jefferson Avenue, Newport News, VA 23606, USA.

\begin{abstract}
I present results from the first lattice QCD calculations of axial-current matrix elements in light nuclei, performed by the NPLQCD collaboration. Precision calculations of these matrix elements, and the subsequent extraction of multi-nucleon axial-current operators, are essential in refining theoretical predictions of the proton-proton fusion cross section, neutrino-nucleus cross sections and $\beta \beta$-decay rates of nuclei. In addition, they are expected to shed light on the phenomenological quenching of $g_{A}$ that is required in nuclear many-body calculations.
\end{abstract}

38th International Conference on High Energy Physics

3-10 August 2016

Chicago, USA

\footnotetext{
* Speaker.

$\dagger$ Presenting on behalf of the NPLQCD collaboration [1]. We thank the Kavli Institute for Theoretical Physics for hospitality during the writing of these proceedings, supported in part by the National Science Foundation under Grant
} No. PHY11-25915 


\section{Introduction}

Axial-current matrix elements play a central role in important aspects of nuclear and particle physics research. For example, the axial-current matrix element in the nucleon is intimately related to the long-range components of the strong nuclear force mediated by pions. In addition, the matrix element between the deuteron and proton-proton continuum at low-energies is responsible for the proton-proton fusion process that initiates the solar-burning cycle. A number of experiments that are currently in production, or are planned for the near-term, depend upon a knowledge of these matrix elements with varying degrees of precision. From a theoretical standpoint, some of the important quantities are easier to determine than others. Lattice QCD calculations have matured to the point where early calculations of the properties and interactions of light nuclei are now being performed, albeit with unphysical values of the light-quark masses and without the electromagnetic interaction. Programs have been established that are on track to perform precision calculations of important low-energy nuclear quantities in the not-so-distant future. Reproducing quantities, such as the excitation spectra of light nuclei and nucleon-nucleon scattering parameters, within the experimental uncertainties will provide verification of the essential elements of LQCD calculations. Analogous verification steps for the nuclear matrix elements of external probes, such as the electroweak interactions, are required for a complete quantification of the uncertainties associated with any suite of LQCD calculations. The spectra of the s-shell nuclei and hypernuclei have been calculated at the $\mathrm{SU}(3)$-symmetric point with a pion mass of $m_{\pi} \sim 805 \mathrm{MeV}$, along with the nucleon-nucleon scattering phase-shifts and parameters in the s-wave and also higher-partial waves. Analogous calculations continue to be performed at lighter quark masses, and calculations in the two-nucleon systems are being performed at the physical point.

The objective of LQCD calculations in multi-nucleon systems is to provide precise and reliable calculations of an array of quantities in few-nucleon systems in order to refine the nuclear forces and interactions that are employed in nuclear many-body calculations of more complex nuclei and systems. In particular, LQCD calculations are starting to constrain multi-nucleon interactions and the correlated two-nucleon interactions with electroweak probes, that are to be propagated into larger nuclei using effective field theory (EFT) techniques. In the case of axial-current matrix elements, it is known that the correlated two-nucleon interactions with axial currents play an important role, and the leading low-energy effects are parameterized by the counterterm $L_{1 A}$ in pionless EFTs, and analogous counterterms in pionful EFTs. In addition to the contributions from $g_{A}$, the protonproton fusion process, tritium $\beta$-decay and neutrino-deuteron scattering and break-up all receive contributions from $L_{1 A}$. Higher energy processes that lie outside the radii of convergence of the low-energy EFTs are more challenging from the hadronic standpoint and reliable theoretical tools for $\mathrm{GeV}$-scale processes remain to be established.

\section{Status of Lattice QCD Calculations of Light Nuclei}

A summary of the nuclear and hypernuclear binding energies obtained by NPLQCD at a pion mass of $m_{\pi} \sim 805 \mathrm{MeV}$ [2] is shown in Fig. 1, from which it is seen that the binding energy per baryon is larger than that found in nature. Consistent a large- $N_{c}$ analysis, the difference in binding between the deuteron and the dineutron is found to be smaller than the splittings to the other states. For 


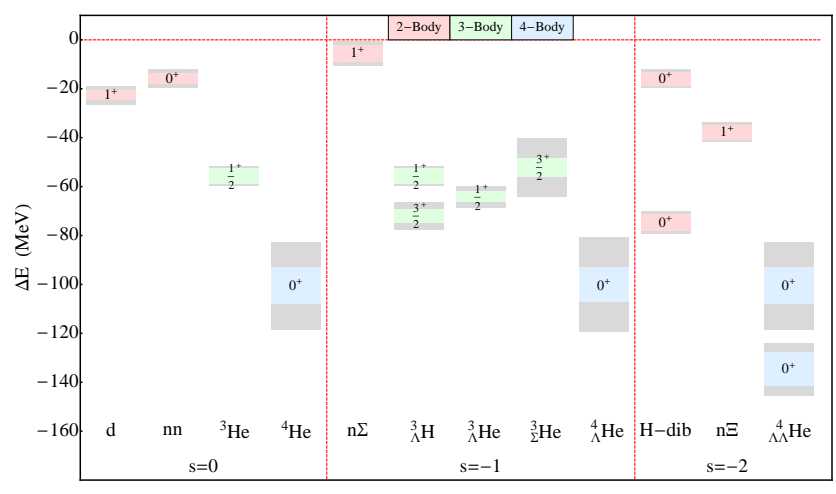

Figure 1: A compilation of the nuclear energy levels at $m_{\pi} \sim 800 \mathrm{MeV}$ with spin and parity $J^{\pi}$ [2].

systems comprised of three baryons, the ground state of ${ }^{3} \mathrm{He}$ (and its isospin partner ${ }^{3} \mathrm{H}$ ) are cleanly identified, as are the ground states, and even one excited hypernucleus. Attaining accurate binding energies for any given light-quark masses will require the inclusion of electromagnetic and isospinbreaking effects. A deeper understanding of the origin and phenomenological interpretation of the calculated binding energies requires a series of nuclear few-body calculations. In particular, it is important to understand the relative contribution from the two-body, three-body, and higher-body contributions to the $A \geq 3$ nuclei and hypernuclei, which can only be accomplished using modern few-body techniques, as recently tackled with the pionless EFT in Refs. $[8,9,10]$. Using the $\alpha$ particle prediction for verification, it was shown that three-nucleon forces are clearly present in the LQCD results. Figure 2 shows the binding energy of the deuteron and dineutron [3, 2, 4] obtained by the PACS collaboration and NPLQCD as a function of the pion mass. ${ }^{1}$ It is exciting to see
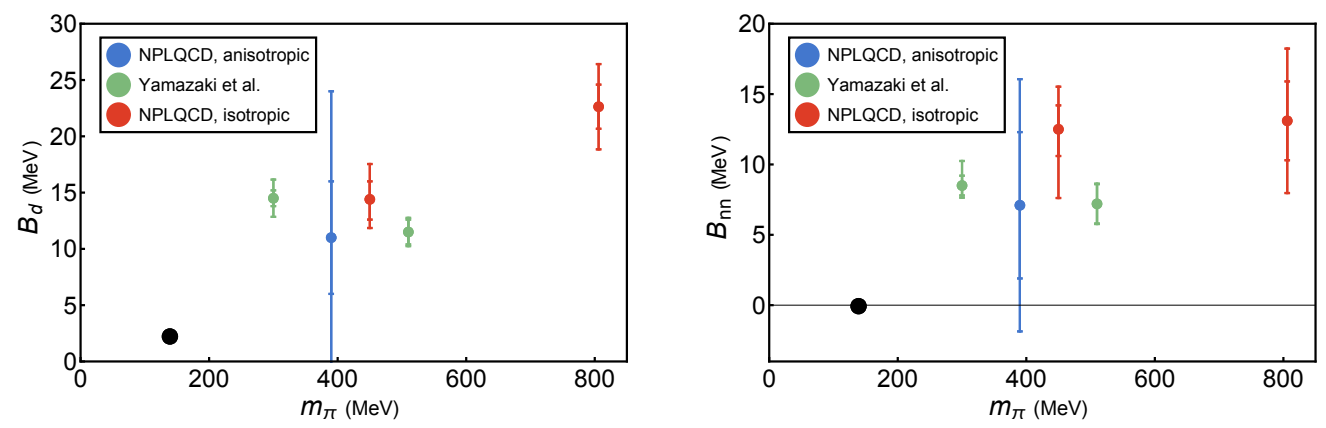

Figure 2: The deuteron (left panel), and dineutron (right panel) binding energies [3, 2, 4, 7$]$.

nuclei emerge from QCD for a range of the light-quark masses, and such calculations are crucial in dissecting and refining the chiral nuclear forces. However, calculations at even lighter pion masses remain critically important.

\section{Magnetic Interactions}

The magnetic moments and polarizabilities of the light nuclei have been calculated at pion masses

\footnotetext{
${ }^{1}$ The HALQCD collaboration does not observe bound states in solutions to the Schrödinger equation with $U_{E}\left(r, r^{\prime}\right) \rightarrow V(r)$ extracted from 4-point Green functions produced with wall-sources. For example, see Ref. [5, 6].
} 
of $m_{\pi} \sim 805 \mathrm{MeV}$ and $\sim 450 \mathrm{MeV}[11,12]$ using background field methods. In addition, the lowenergy cross section for the simplest radiative capture process, $n p \rightarrow d \gamma$, has been calculated [14]. A number of interesting features and observations emerge from these calculations, despite the unphysical masses of the light quarks, and extrapolations to the physical point have been accomplished. Perhaps the most interesting observation is that when the moments are given in units defined by the baryon mass, they are relatively insensitive to the light-quark masses, with little variation observed between $m_{\pi} \sim 805 \mathrm{MeV}$ and the physical point, as shown in Figure 3. Further,
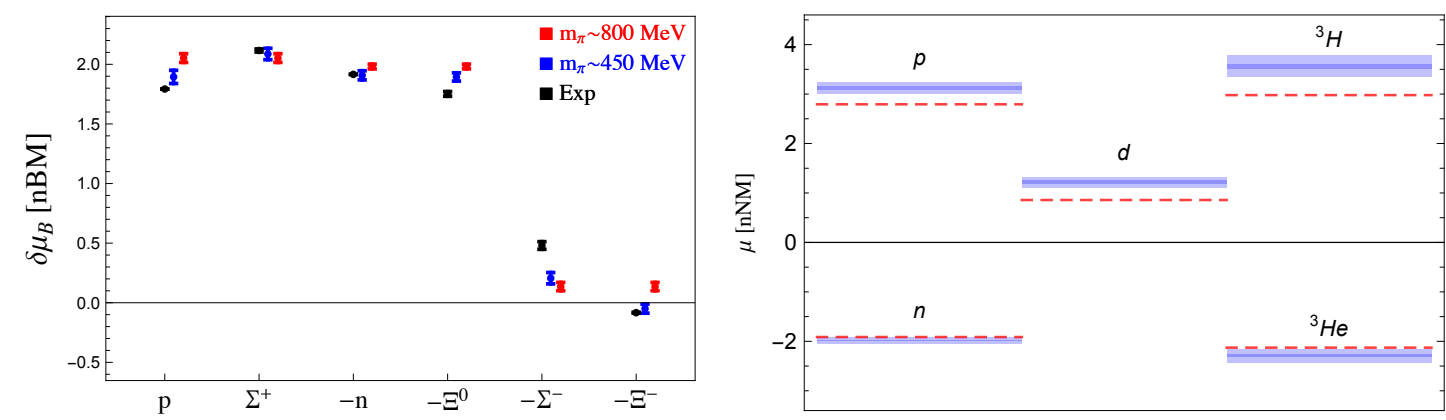

Figure 3: The anomalous magnetic moments of the lowest-lying octet of baryons [13] (left panel) in units of natural Baryon Magnetons, and the magnetic moments of the light nuclei in units of natural Nuclear Magnetons calculated at $m_{\pi} \sim 805 \mathrm{MeV}$ compared with experiment $[11,12]$ (right panel).

the deviations from predictions of the naive single-particle shell model at the heavier quark masses are consistent with the corresponding experimental values, within the uncertainties of the calculations.

The very low-energy radiative-capture process $n p \rightarrow d \gamma$ is dominated by the M1 matrix element, which receives most of its contribution from the nucleon isovector magnetic moment and from correlated two-nucleon interactions with the magnetic field (meson-exchange currents). By performing LQCD calculations at the heavier quark masses [14], the counterterm in the low-energy EFT describing $n p \rightarrow d \gamma$ has been determined and extrapolated to the physical point. Like the magnetic moments themselves, it is found to be quite insensitive to the masses of the light quarks within the uncertainties of the calculations. Using the experimentally measured scattering parameters and the LQCD determined counterterm, the post-dicted $n p \rightarrow d \gamma$ cross section is found to agree with experiment within the uncertainties.

\section{Axial-Current Matrix Elements}

Important low-energy axial-current matrix elements are starting to be determined from LQCD calculations, using methods analogous to those used to determine magnetic matrix elements. Axial background fields are used to generate light-quark propagators, which in turn are used to generate the relevant axial-current matrix elements. At present, calculations are performed at unphysical values of the light quark masses and extrapolated to the physical point with EFTs. Further, only one spacetime volume with one discretization is employed. Once the appropriate renormalization factor that relates the lattice axial current to that in the continuum is employed, the value of $g_{A}$ in the proton is recovered at $m_{\pi} \sim 805 \mathrm{MeV}$ within the uncertainties of the calculations. 
The leading matrix element contributing to low-energy proton-proton fusion, the nuclear reaction that initiates the solar-burning cycle, has been calculated at $m_{\pi} \sim 805 \mathrm{MeV}$, leading to a constraint on the higher-order isovector counterterm in the pionless EFT, $L_{1 A}$, as shown in Figure 4. As the low-energy operator structure for this process is the same as for $n p \rightarrow d \gamma$ and the
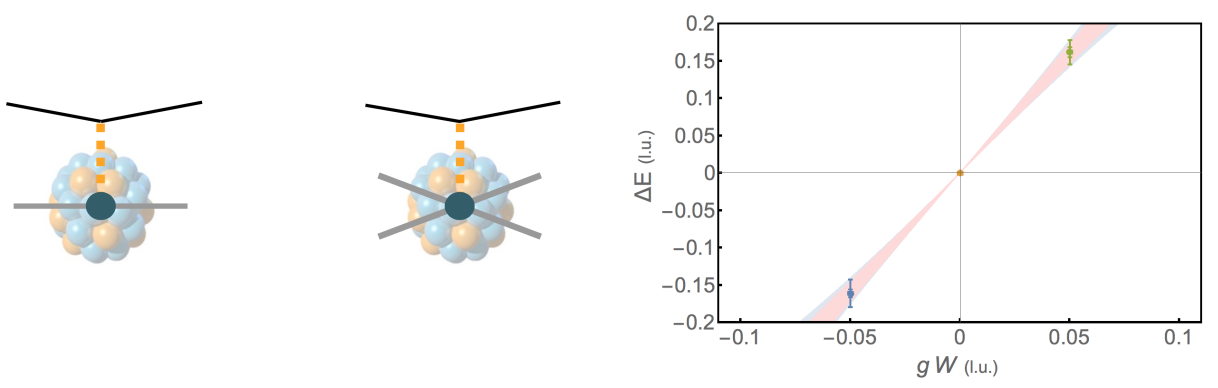

Figure 4: The left panel shows a cartoon of the single-nucleon and correlated two-nucleon axial-current interactions, while the right panel shows preliminary energy splittings between the two lowest-lying eigenstates in the $J_{z}=I_{z}=0$ two-nucleon sector as a function of the background axial field.

matrix element in the nucleon, $g_{A}$, is also fairly insensitive to the light-quark masses over this interval, a value and systematic uncertainty has been assigned at the physical point. Clearly, this is a first calculation of this process, and calculations over a range of pion masses and at the physical point are required. Figure 4 shows the energy splittings between the two lowest-lying eigenstates in the $J_{z}=I_{z}=0$ two-nucleon sector as a function of the background axial field, which can be used to constrain $L_{1 A}$. The Gamow-Teller matrix element contributing to the $\beta$-decay of tritium can also be determined with axial background-field calculations. Such calculations at $m_{\pi} \sim 805 \mathrm{MeV}$ lead to a value of $g_{A}\left({ }^{3} H\right)$ that is close to that of the proton, and consequently, by isospin symmetry, a value of the Gamow-Teller matrix element that is consistent with experiment. This is further indication of a weak pion mass dependence of the higher order contributions in the low-energy EFT.

Finally, the background-field technique enables a determination of the isotensor axial polarizability of the dinucleon spin-singlet system, which contains the matrix element for $n n \rightarrow p p$ transitions relevant for $\beta \beta$-decay processes in nuclei. The axial polarizability contributes to energy shifts that depend quadratically on the background axial field strength.

\section{Post-Presentation Progress}

Our initial work on axial-current matrix elements was recently completed [15]. The Gamow-Teller matrix element contributing to tritium $\beta$-decay was found to be $\mathrm{GT}=0.979(03)(10)$, which should be compared to the experimental value of $\mathrm{GT}^{\mathrm{expt}}=0.9511(13)$. The $\mathrm{S}$-factor for pp-fusion, in the absence of electromagnetism and isospin breaking, was found to be $\Lambda(0)=2.6585(06)(72)(25)$, assuming that the correlated two-nucleon interactions are independent of the light-quark masses and assigning a systematic uncertainty based upon what is observed in $n p \rightarrow d \gamma$, leading to a value of $L_{1 A}=3.9(0.1)(1.0)(0.3)(0.9) \mathrm{fm}^{3}$. This is to be compared with the currently accepted value of $\Lambda(0)=2.652(2)$. These results are the first of such calculations, and demonstrate that, with sufficient computing resources, axial-current matrix elements can be determined with precision from ongoing and future LQCD calculations. 


\section{Summary and Outlook}

Results of the first Lattice Quantum Chromodynamics calculations of matrix elements of the axial current in light nuclei have been presented. In particular, first calculations of the Gamow-Teller matrix element contributing to the $\beta$-decay of tritium, and the low-energy cross-section for ppfusion have been performed by the NPLQCD collaboration at a pion mass of $m_{\pi} \sim 805 \mathrm{MeV}$. They yield results that are consistent with experiment and phenomenology, respectively, under the assumption of mild quark-mass dependence of the correlated two-nucleon contributions, consistent with what is found for $n p \rightarrow d \gamma$. Finally, this work paves the way for axial-current form factors of light nuclei to be addressed with LQCD.

\section{References}

[1] http://www.ecm.ub.es/ assum/NPLQCD/index.html

[2] S. R. Beane et al. [NPLQCD Collaboration], Phys. Rev. D 87, no. 3, 034506 (2013) doi:10.1103/PhysRevD.87.034506 [arXiv:1206.5219 [hep-lat]].

[3] S. R. Beane et al. [NPLQCD Collaboration], Phys. Rev. D 85, 054511 (2012) doi:10.1103/PhysRevD.85.054511 [arXiv:1109.2889 [hep-lat]].

[4] T. Yamazaki, K. i. Ishikawa, Y. Kuramashi and A. Ukawa, Phys. Rev. D 92, no. 1, 014501 (2015) doi:10.1103/PhysRevD.92.014501 [arXiv:1502.04182 [hep-lat]].

[5] S. Aoki, Eur. Phys. J. A 49, 81 (2013) doi:10.1140/epja/i2013-13081-0 [arXiv:1309.4150 [hep-lat]].

[6] T. Iritani et al., JHEP 1610, 101 (2016) doi:10.1007/JHEP10(2016)101 [arXiv:1607.06371 [hep-lat]].

[7] K. Orginos, A. Parreno, M. J. Savage, S. R. Beane, E. Chang and W. Detmold [NPLQCD Collaboration], Phys. Rev. D 92, no. 11, 114512 (2015) doi:10.1103/PhysRevD.92.114512 [arXiv:1508.07583 [hep-lat]].

[8] N. Barnea, L. Contessi, D. Gazit, F. Pederiva and U. van Kolck, Phys. Rev. Lett. 114 (2015) no.5, 052501 doi:10.1103/PhysRevLett.114.052501 [arXiv:1311.4966 [nucl-th]].

[9] U. van Kolck, Few Body Syst. 56, no. 11-12, 745 (2015) doi:10.1007/s00601-015-0980-1 [arXiv:1505.06323 [nucl-th]].

[10] J. Kirscher, Int. J. Mod. Phys. E 25, no. 05, 1641001 (2016) doi:10.1142/S0218301316410019 [arXiv:1509.07697 [nucl-th]].

[11] S. R. Beane et al. [NPLQCD Collaboration], Phys. Rev. Lett. 113, no. 25, 252001 (2014) doi:10.1103/PhysRevLett.113.252001 [arXiv:1409.3556 [hep-lat]].

[12] E. Chang et al. [NPLQCD Collaboration], Phys. Rev. D 92, no. 11, 114502 (2015) doi:10.1103/PhysRevD.92.114502 [arXiv:1506.05518 [hep-lat]].

[13] A. Parreno, M. J. Savage, B. C. Tiburzi, J. Wilhelm, E. Chang, W. Detmold and K. Orginos [NPLQCD Collaboration], arXiv:1609.03985 [hep-lat].

[14] S. R. Beane et al. [NPLQCD Collaboration], Phys. Rev. Lett. 115, no. 13, 132001 (2015) doi:10.1103/PhysRevLett.115.132001 [arXiv:1505.02422 [hep-lat]].

[15] M. J. Savage et al. [NPLQCD Collaboration], arXiv:1610.04545 [hep-lat]. 